\title{
Induction of Anthocyanin Accumulation in Crabapple (Malus cv.) Leaves by Low Temperatures
}

\author{
Ji Tian', Zhen-yun Han', Li-ru Zhang', Ting-Ting Song, Jie Zhang, \\ Jin-Yan Li, and Yuncong Yao ${ }^{2}$ \\ Department of Plant Science and Technology, Beijing Key Laboratory for \\ Agricultural Application and New Technique, Beijing University of \\ Agriculture, Beijing 102206, China
}

Additional index words. MBW complex, Malus crabapple, anthocyanins, leaves, transcription factor, low temperature

\begin{abstract}
Anthocyanins are protective pigments that accumulate in plant organs such as fruits and leaves, and are nutritionally valuable components of the human diet. There is thus considerable interest in the factors that regulate synthesis. Malus crabapple leaves are rich sources of these compounds, and in this study we analyzed leaf coloration, anthocyanin levels, and the expression levels of anthocyanin biosynthetic and regulatory genes in three crabapple cultivars (Royalty, Prairifire, and Flame) following various temperature treatments. We found that low temperatures (LTs) promoted anthocyanin accumulation in 'Royalty' and 'Prairifire', leading to red leaves, but not in 'Flame', which accumulated abundant colorless flavonols and retained green colored leaves. Quantitative reverse transcript PCR (RT-PCR) analyses indicated that the expression of several anthocyanin biosynthetic genes was induced by LTs, as were members of the R2R3-MYB, basic helix-loop-helix (bHLH) and WD40 transcription factor families that are thought to act in a complex. We propose that anthocyanin biosynthesis is differentially regulated in the three cultivars by LTs via the expression of members of this anthocyanin regulatory complex.
\end{abstract}

Flavonoids are generated via a branched biosynthetic pathway that yields both colorless compounds (e.g., flavonols) and colored pigments, such as anthocyanins, polymeric phlobaphenes, and proanthocyanidins (Koes et al., 2005). Flavonoids accumulate in all organs and tissues, although their concentrations are regulated by development stage and the environmental conditions (Hichri et al., 2011). Moreover, as they have antioxidant activities that can help prevent cancer, as well as cardiovascular and neurodegenerative

Received for publication 31 Dec. 2014. Accepted for publication 27 Feb. 2015

We thank the Fruit Tree Key Laboratory at the Beijing University of Agriculture, and the Beijing Key Laboratory for Agricultural Application and New Technique for providing experimental facilities. We are grateful to all technicians in the BUA Crabapple Germplasm Resource Garden. We also thank PlantScribe (www.plantscribe.com) for carefully editing this manuscript. Financial support was provided by National Science and Technology Support Program (2013BAD02B01-4), the Project of Construction of Innovative Teams and Teacher Career Development for Universities and Colleges under Beijing Municipality (IDHT20140509), the National Natural Science Foundation of China (31301762), and Beijing Municipal Commission of Education Science and Technology Promotion Plan (PXM2014-014207-000081).

The authors declare no competing financial interests.

${ }^{1}$ Contributed equally to this work.

${ }^{2}$ To whom reprint requests should be addressed; e-mail yaoyc_20@126.com. diseases, they are therefore beneficial to human health (Boudet, 2007; Martin, 2013). Consequently, the flavonoid biosynthetic pathway leading to anthocyanins via the phenylpropanoid pathway has been extensively investigated and it has been shown qualitatively (Winkel-Shirley, 2001) that the profile of specific anthocyanins in an organ varies between species/varieties, whereas environmental factors influence their concentrations (Moreau et al., 2012; Pfeiffer et al., 2006; Rowan et al., 2009).

The regulation of the expression of structural genes involved in anthocyanin synthesis appears to be tightly spatially and temporally controlled during plant development, and involves a ternary complex of transcription factors (TFs) from the R2R3-MYB, bHLH, and WD40 classes (Hichri et al., 2011). The $\mathrm{MYB} / \mathrm{bHLH} / \mathrm{WDR}$ (MBW) complex is thought to recognize and bind to response elements in promoter regions of the late biosynthetic genes (LBGs) of the anthocyanin pathway, thereby promoting anthocyanin production (Baudry et al., 2004). Ternary complexes have been identified in monocots and dicotyledonous plants species (Feller et al., 2011).

Several studies have also described the flavonoid biosynthetic pathway in Malus crabapple (Fig. 1), which accumulates high levels of anthocyanins in its leaves and flowers (Deluc, 2006; Henry-Kirk et al., 2012; Schaart et al., 2013). Indeed, crabapple leaves are used as a raw material for the extraction of antioxidants for use as food additives (Tian et al., 2011) and crabapple leaf tea is a popular health beverage in many parts of Asia. Anthocyanin accumulation in Malus crabapple leaves has been shown to be controlled by the regulation of the flavonoid/ anthocyanin biosynthesis by the MBW complex in response to environmental $\mathrm{pH}$ values (Zhang et al., 2014). Moreover, anthocyanin biosynthesis can also be accelerated by high light levels, LTs, or both. In late autumn, mature Malus crabapple fruits turn red and leaves "blush" before undergoing abscission. In apple (Malus domestica), the expression of MYB activator genes, especially $M d M Y B 10$, is downregulated in fruits grown under natural or artificially hot conditions compared with fruits grown under temperate conditions (Lin-Wang et al., 2011). In addition, viral vector-mediated $M d b H L H 3$ overexpression was shown to promote red pigmentation in apple fruits, and this phenomenon was further enhanced by LT conditions (Xie et al., 2012). Previous studies of the MBW complex have revealed the expression of complex members, specifically bHLHs and MYBs, is modulated by temperature, suggesting a role for the MBW regulatory complex in environmentally influenced anthocyanins production (Xie et al., 2012). However, most of these studies have involved fruits and the molecular mechanisms that govern anthocyanins accumulation in response to temperature changes in leaves remain unknown.

Although only one gene, McMYB10, has been shown to regulate anthocyanin biosynthesis in Malus crabapple (Jiang et al., 2014), it has been established that the formation and accumulation of anthocyanins in Malus crabapple leaves is affected by $\mathrm{pH}$ environmental factor (Zhang et al., 2014). With the increased mean global temperatures, plant color variation is attracting more and more

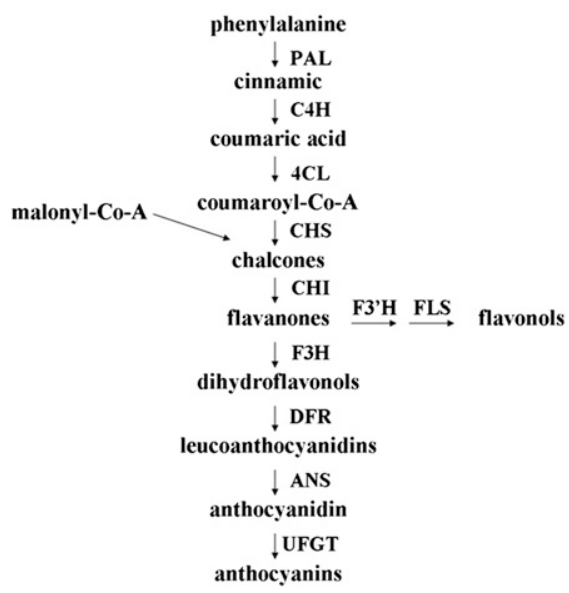

Fig. 1. Scheme of the anthocyanins biosynthetic pathway. Enzymes for each step are indicated as follows: PAL, phenylalanine ammonium lyase-1; C4H, cinnamate-4-hydroxylase; CHS, chalcone synthase; $\mathrm{CHI}$, chalcone isomerase; F3H, flavanone 3-hydroxylase; DFR, dihydroflavonol 4-reductase; ANS, anthocyanidin synthase; GT, uridine diphosphate glucose (UDPG)-flavonoid glucosyl transferase. 
attention and research. In this current study, we investigated how temperature affects the content of flavonoids, and especially anthocyanins, in the leaves of Malus crabapple cultivars in response to different temperature treatments. Three typical crabapple cultivars were chosen based on different leaf coloration patterns. The two extreme color cultivars, Royalty and Flame, have purple and green leaves, respectively, whereas the cultivar Radiant has red young leaves and green mature leaves. The involvement of LTs in the induction of anthocyanin accumulation is discussed in different crabapple cultivars.

\section{Materials and Methods}

Plant materials and experimental treatments. Tissue culture plantlets of the Malus crabapple cultivars Flame (both young and mature leaves are green), Prairifire (young leaves are orange to red and mature leaves are green), and Royalty (both young and mature leaves are red to purple in color) were preserved at the tissue culture center at the Beijing University of Agriculture. Culture conditions were as follows: temperature $23 \pm 2{ }^{\circ} \mathrm{C}$, humidity $60 \%$ to $70 \%$, light intensity $1800-2000 \mathrm{~lx}$ with a $16-\mathrm{h}$ light/ 8 -h dark cycle. The buds of these three cultivars were cultured on Murashige and Skoog (MS) medium supplemented with $2.2 \mu \mathrm{M}$ 6-benzylaminopurine (6-BA) and $0.5 \mu \mathrm{M}$ 1-naphthaleneacetic acid (NAA) for $30 \mathrm{~d}$ to induce leaf reproduction before temperature treatments. Temperature treatments involved
$30 \mathrm{~d}$ leaves to $30^{\circ} \mathrm{C}$ or $16^{\circ} \mathrm{C}$ for 4 or $8 \mathrm{~d}$, after which leaves were frozen in liquid nitrogen and stored at $-80{ }^{\circ} \mathrm{C}$ until further use.

Measurement of flavonoid concentration and leaf color. Quantification of pigments was performed by high-performance liquid chromatography (HPLC). The color variables of each crabapple leaf sample were measured immediately after harvest. $\mathrm{L}^{*}, \mathrm{a}^{*}$, and $b^{*}$ values were measured randomly from the upper leaf surface using a Konica Minolta CR-400 Chroma Meter (Minolta, Japan). Flavonoid concentration and leaf color were measured as previously described (Zhang et al., 2014).

Cloning and sequence analysis of fulllength McbHLH3, McbHLH33, and McTTG1 $c D N A s$. Total RNA was isolated from young leaves of 'Royalty' using a guanidine thiocyanate solution (Chomczynski and Sacchi, 2006). cDNAs were prepared from $1 \mu \mathrm{g}$ of total RNA using Reverse Transcriptase M-MLV First cDNA Synthesis Kit (Takara, Japan) and the targeted specific cDNAs, amplified by PCR using the primers listed in Table 1, were cloned into the pMD-19 vector (Takara, Japan) for sequencing. The PCR conditions were $5 \mathrm{~min}$ at $95{ }^{\circ} \mathrm{C}$, followed by 35 cycles of $30 \mathrm{~s}$ at $95{ }^{\circ} \mathrm{C}, 30 \mathrm{~s}$ at $60^{\circ} \mathrm{C}$, and $2 \mathrm{~min}$ at $72^{\circ} \mathrm{C}$, followed by $7 \mathrm{~min}$ at $72{ }^{\circ} \mathrm{C}$. Comparison and analysis of the sequences were performed using the advanced basic local alignment search tool (BLAST) at the National Center for Biotechnological Information (http://www.ncbi. nlm.nih.gov). The full-length DNA and protein sequences were aligned using DANMAN 5.2.2 (Lynnon Biosoft, San Francisco, CA). Phylogenetic and molecular evolutionary analyses were conducted with MEGA version 4.0, using a minimum evolution phylogeny test with a 1000 bootstrap replicates (Kumar et al., 2004).

Real-time quantitative PCR expression analysis. Total leaf RNA was extracted using an RNA extract kit (Aidlab, Beijing, China) according to the manufacturer's instructions. DNase I (Takara, Japan) was added to remove genomic DNA, and the samples were then subjected to cDNA synthesis using the Access RT-PCR System (Promega, Madison, WI) according to the manufacturer's instructions. The expression levels of anthocyanin biosynthetic genes and TFs were analyzed using quantitative real-time PCR (RT-qPCR) with SYBR Green qPCR Mix (Takara, Japan) and the Bio-Rad CFX96 Real-Time PCR System (BIO-RAD, Houston, TX), according to the manufacturer's instructions. Primers were designed using NCBI Primer BLAST (www.ncbi.nlm.nih.gov/tools/primer-blast) and are listed in Table 1. qPCR analysis was carried out in a total volume of $20 \mu \mathrm{L}$ containing $9 \mu \mathrm{L}$ of $2 \times$ SYBR Green qPCR Mix (Takara, Japan), $0.1 \mu \mathrm{M}$ specific primers (each), and $100 \mathrm{ng}$ of template cDNA. The reaction mixtures were heated to $95^{\circ} \mathrm{C}$ for $30 \mathrm{~s}$, followed by 39 cycles at $95{ }^{\circ} \mathrm{C}$ for $10 \mathrm{~s}, 59^{\circ} \mathrm{C}$ for $15 \mathrm{~s}$, and $72{ }^{\circ} \mathrm{C}$ for $30 \mathrm{~s}$. A melting curve was generated for each sample at the end of each run to ensure the purity of the amplified products. Transcript levels were determined by relative quantification

Table 1. Specific primer sequences that are used in this study.

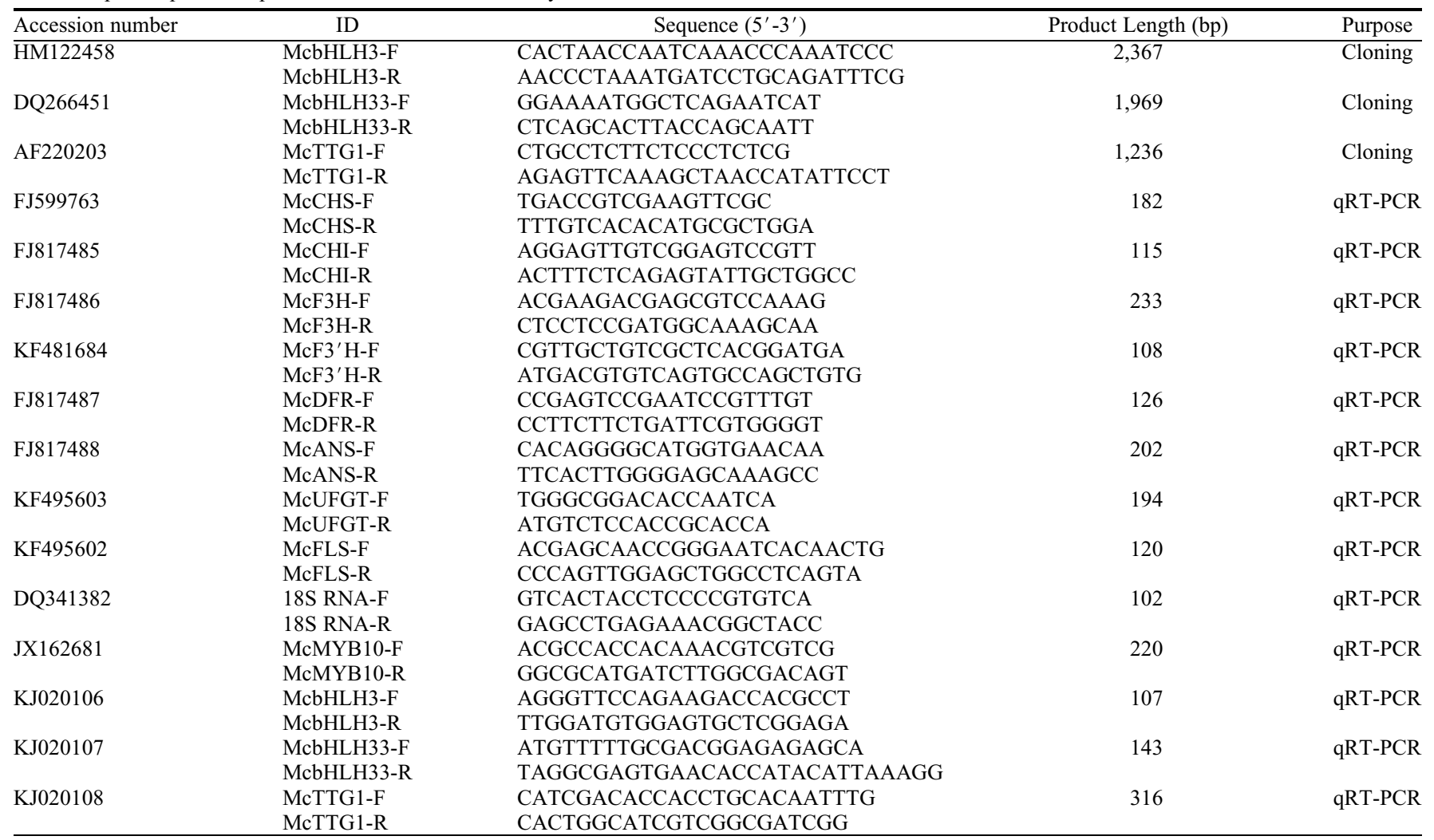

qRT-PCR, quantitative reverse transcript polymerase chain reaction. 
using the Malus 18S ribosomal RNA gene as the internal control using the $2^{(-\Delta \Delta C t)}$ method. The final data presented here represent the averages of at least two technical and three biological replicates.

Statistical analysis. All data were analyzed using one-way analysis of variance (ANOVA) followed by Duncan's SSR test (shortest significant ranges) to compare differences among the experimental sites at $P<$ 0.05 [Microsoft Excel 2003 and Data Processing System (DPS) software 7.05].

\section{Results}

Effect of temperature on foliage coloration. As shown in Figure 2A, a visible red color appeared on the entire upper surface of 'Royalty' leaves after $4 \mathrm{~d}$ of LT treatment at $16{ }^{\circ} \mathrm{C}$, and this became a deeper red color after $8 \mathrm{~d}$. In contrast, red coloration was only visible on the upper portion of the leaves of 'Prairifire', even after $8 \mathrm{~d}$ of LT treatment, whereas no red color was observed in leaves of the crabapple cultivar Flame after the different temperature treatments.

The variations in leaf color during different temperature treatments were quantified using color index for red grape (CIRG) values, and an increase with time was observed under LT conditions $\left(16^{\circ} \mathrm{C}\right)$ in 'Royalty' and 'Prairifire' (Fig. 2B). Although the leaf color of 'Royalty' changed from green to red after $4 \mathrm{~d}$, the leaf color of 'Prairifire' exhibited this change only after $8 \mathrm{~d}$ of LT treatment (Fig. 2A). The CIRG values of 'Flame' were relatively low level compared with the other two cultivars (Fig. 2B).

We then measured flavonoid composition and accumulation in leaves of the three different cultivars after exposure to different temperatures by HPLC (Fig. 2C). Low temperatures induced and high temperature repressed anthocyanin accumulation in the leaves of 'Royalty', where concentrations were notably higher than that in the other two cultivars. The change of anthocyanin accumulation in the leaves of 'Prairifire' showed a similar trend to 'Royalty', but the abundance of anthocyanin was significantly lower, whereas anthocyanins were undetectable in 'Flame' leaves. The concentration of flavonol in 'Flame' also decreased slightly with LT treatments, which is consistent with the variation trend of anthocyanin accumulation in 'Royalty'. Low temperature conditions resulted in higher levels of flavones in all three cultivars, with a pattern among the cultivars similar to that of anthocyanins, although the levels were lower than those of anthocyanins and flavonol. Taken together, these results suggest that temperature can trigger pigmentation variations, which correlate with a variation in flavonoid component.

Cloning of transcription factors with temperature response. The open reading frames (ORFs) of the McbHLH3 (KJ020106), McbHLH33 (KJ020107), and McTTG1 (KJ020108) TF genes were isolated from crabapple. The 2130 base pair (bp), $1956 \mathrm{bp}$,

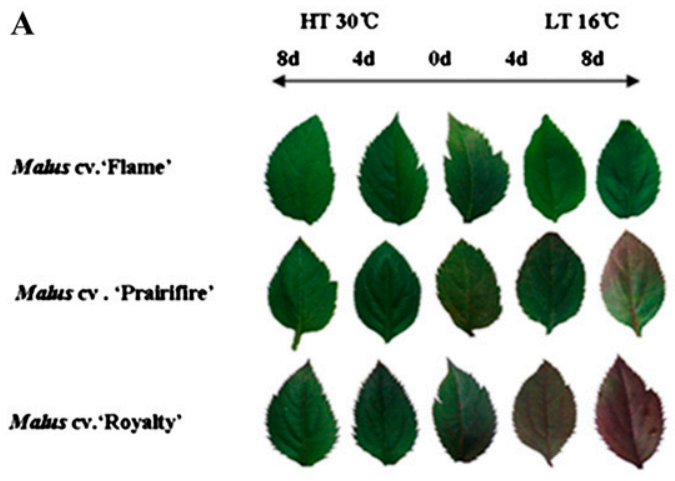

B

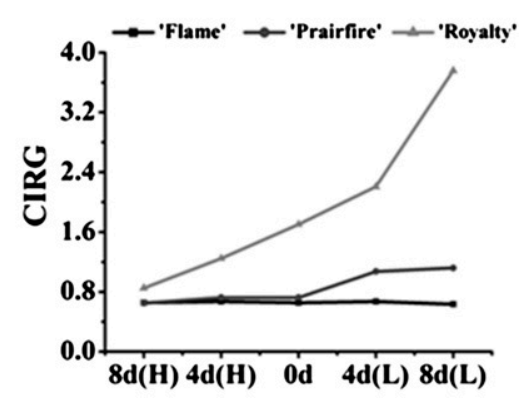

C
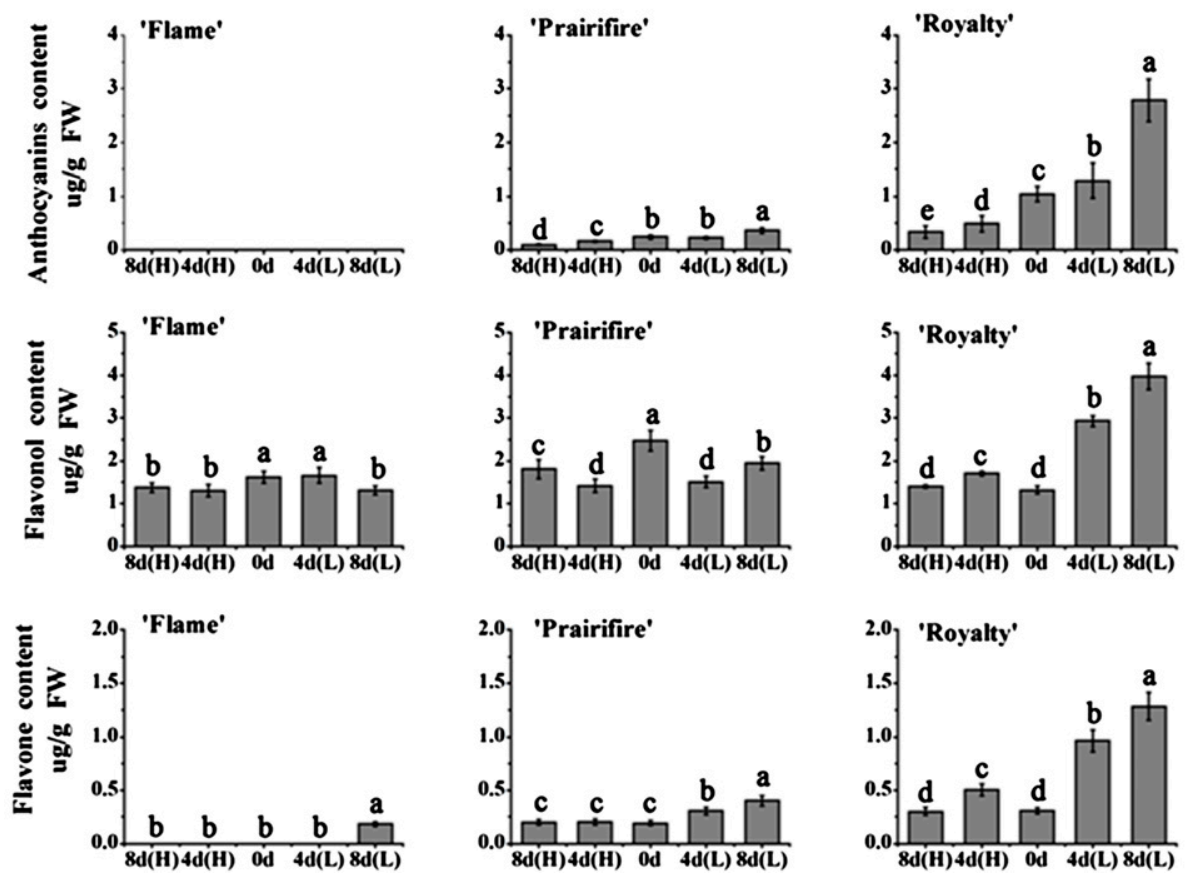

Fig. 2. Different temperature treatments can alter anthocyanin accumulation in crabapple foliage. (A) Low temperature induces the accumulation of red color in Malus crabapple foliage except the 'Flame'. HT stands for high temperature, LT stands for low temperature. The horizontal ordinate shows the days after temperature treatment. (B) CIRG values determination during temperature treatment of three different cultivars. (C) HPLC analysis the variation of flavonoids in crabapple leaves under different temperature treatments. 
and 1029 bp full-length cDNAs of McbHLH3, $M c b H L H 33$, and McTTG1, respectively, are predicted to encode proteins 709,651 , and 342 amino acid residues, respectively. These deduced protein sequences share $99.7 \%, 98 \%$, and $99.4 \%$ sequence similarity to MdbHLH3, MdbHLH33, and MdTTG1, respectively, which are homologs from domesticated apple. Structural analysis further showed that McbHLH3, McbHLH33, and McTTG1 contain conserved domains that are typical features of each family (Fig. 3). N-terminal
A

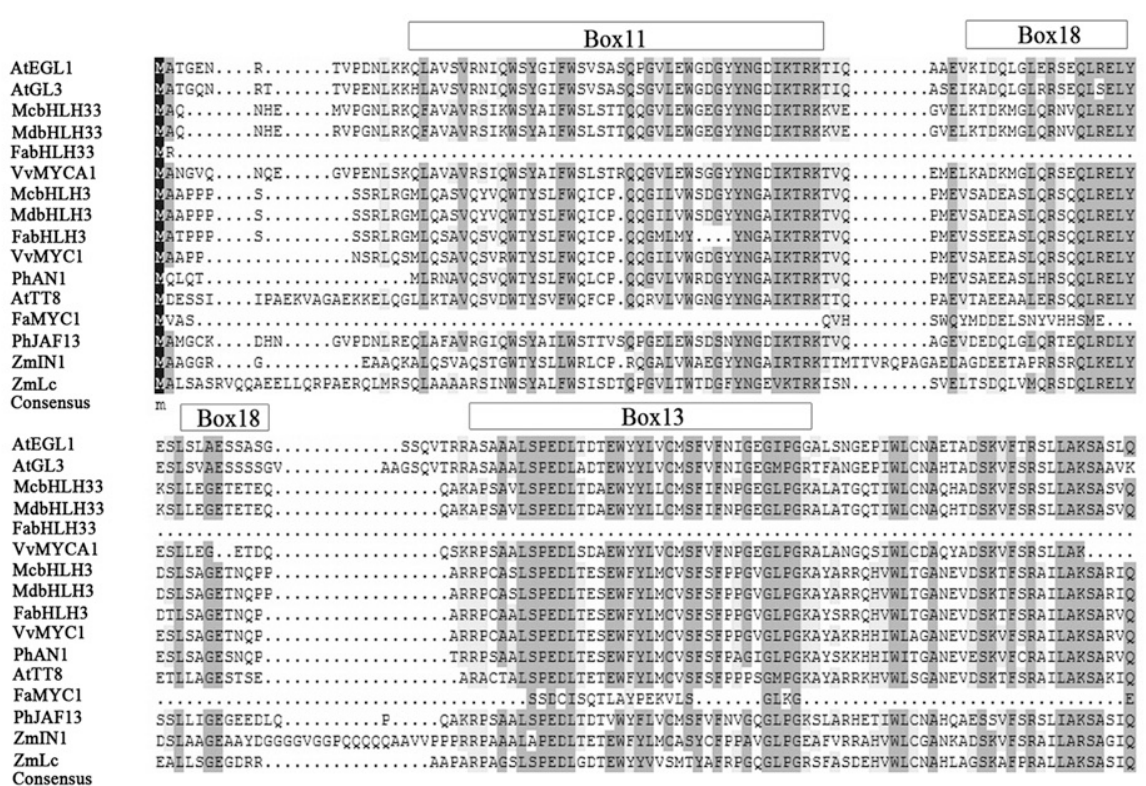

AtEGL1

AtGL3

McbHLH33

MabHLH33

VvMYCA

$\mathrm{McbHLH} 3$

FabHLH3

PhAN1

AtTr

FaMYCl

ZmIN1

ZmLc

Basic Helix1 Loop Helix2

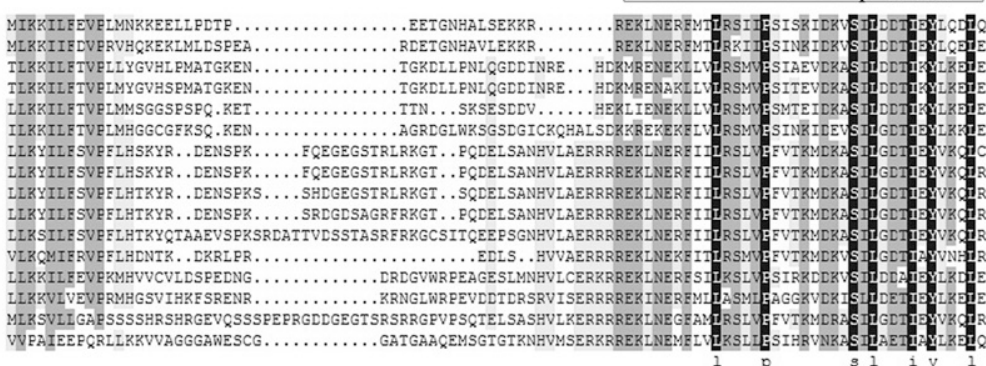

487

500

500

111
499

526

526

516

540
521

409

215

484
514

B

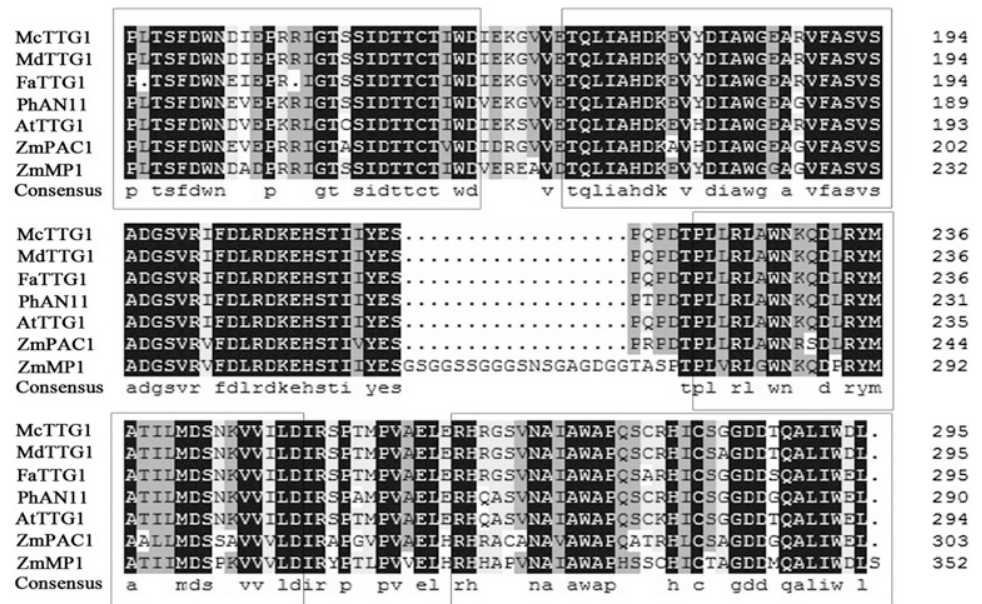

Fig. 3. McbHLH3, McbHLH33, and McTTG1 share homology at the typical domains with anthocyanin regulators from other species. (A) The structure of the basic helix-loop-helix (bHLH) binding domain and N-terminus of the same bHLH subset showing regions (boxes 11, 18, and 13) conserved within the bHLH IIIf clade (according to Heim et al., 2003). (B) Sequence comparison of selected TTG1-like WD40 proteins, the WD-repeat domains were marked by four open boxes. Identical residues are shown in black, conserved residues in dark gray, and similar residues in light gray. The GenBank accession numbers of the proteins are as follows: AtTT8 (Arabidopsis thaliana, CAC14865); PhAN1 (Petunia hybrid, AAG25928); MdbHLH3 (Malus domestica, CN934367); MdbHLH33 (Malus domestica, DQ266451); VvMYC1 (Vitis vinifera, ACC68685); AtGL3 (Arabidopsis thaliana, NP 680372); FabHLH3, (Fragaria ananassa, JQ989284); FabHLH3, (Fragaria ananassa, JQ989285); AtEGL3 (Arabidopsis thaliana, NP_176552); PhJAF13 (Petunia hybrid, AAC39455); FabHLH33 (Fragaria ananassa, JQ989286); FaMYC1 (Fragaria ananassa, JQ989283); VvMYCA1 (Vitis vinifera, ABM92332); ZmLc (Zea mays, AAA33504); ZmIN1 (Zea mays, AAB03841); McbHLH3 (Malus crabapple, KJ020106); McbHLH33 (Malus crabapple, KJ020107); FaTTG1 (Fragaria ananassa, JQ989287); ZmPAC1 (Zea mays, AAM76742); AtTTG1 (Arabidopsis thaliana, CAB45372); PhAN11 (Petunia hybrid, AAC18914); MdTTG1 (Malus domestica, ADI58760); ZmmP1 (Zea mays, AAR01949); VvWDR1 (Vitis vinifera, ABF6662); McTTG1 (Malus crabapple, KJ020108). 
domains identified as important for MYB interaction are present in both McbHLH3 and McbHLH33, and the WD40 repeat domain is present in McTTG1 (Heim et al., 2003). Phylogenetic analyses showed that each of the three predicted proteins from crabapple clusters on the same branch as the corresponding homolog from domesticated apple, suggesting that they may have similar functions in regulating anthocyanin biosynthesis (Fig. 4).

Relative expression of anthocyanin biosynthetic genes under LT condition. To correlate leaf color with potential molecular mechanisms that mediate anthocyanin formation, the levels of transcripts encoding eight anthocyanin biosynthetic genes in Figure 1 were compared in the three cultivars using real-time qPCR. The transcript levels of the key anthocyanin biosynthetic genes $M c C H S, M c F 3 H$, and $M c D F R$ were consistent with the observed anthocyanin accumulation, with the highest transcript levels being present in 'Royalty' after $8 \mathrm{~d}$ at $16{ }^{\circ} \mathrm{C}$. We also noted a correlation between the expression of $M c F 3^{\prime} H$ and flavonol accumulation (Fig. 5A and B).

We observed that the transcript abundance of $M c C H S, M c F 3 H$, and $M c D F R$ increased in response to $\mathrm{LT}$, and we propose that the high expression levels of these biosynthesis genes in 'Royalty' and 'Prairifire' may be responsible for the deep red color formation. However, as flavonol is the main flavonoid compound in 'Flame', and the expression of $M c F 3^{\prime} H$ decreases in 'Flame' under LTs, this gene may be responsible for flavonol production (Figs. $2 \mathrm{C}$ and $5 \mathrm{~A}$ ).

Relative expression of anthocyaninregulated transcription factors under $L T$ condition. To investigate the molecular regulatory mechanisms, and specifically the involvement of MBW complex, in the LT induction of anthocyanin accumulation, the transcript levels of four TFs (McMYB10, McbHLH3, McbHLH33, and McTTG1) were assessed using real-time qPCR. The expression of all four genes increased over the course of the LT treatment in 'Royalty' and 'Prairifire', a trend that generally mirrors the patterns of anthocyanin abundance in these two cultivars. Interestingly, the expression of McbHLH3, McbHLH33, and McTTG1 was higher in 'Flame' when the treatment temperature was $16{ }^{\circ} \mathrm{C}$, and the pattern of McMYB 10 transcript abundance was similar to that of the flavonol content in 'Flame' leaves. To conclude, the expression patterns of McMYB10, McbHLH3, McbHLH33, and $M c T T G 1$, and specially McbHLH3, support their proposed involvement in mediating the upregulation of anthocyanin biosynthesis in response to LTs (Fig. 5C).

A relative analysis revealed a correlation between anthocyanin biosynthetic genes and anthocyanin-regulated TFs (Tables 2-4). The expression of McMYB10, McbHLH3, McbHLH33, and McTTG1 showed a positive correlation with that of $M c C H S(0.88,0.79$, $0.5,0.59)$, and a negative correlation with that of McDFR $(-0.37,-0.5,-0.74,-0.79)$ in 'Flame'. The expression of McMYB10, McbHLH3, McbHLH33, and McTTG1 was particularly associated with the expression of anthocyanin biosynthetic genes in the sequential steps in the pathways from $\mathrm{McCHS}$ to $M c A N S$ in 'Prairifire'. In addition, the expression of $M c C H S(0.92,0.97,0.96,0.7)$, $M c F 3 H(0.83,0.90,0.85,0.85)$, and $M c D F R$ $(0.83,0.90,0.84,0.88)$ showed a strong correlation with the anthocyanin-related TFs (McMYB10, McbHLH3, McbHLH33, and McTTG1) in 'Royalty'. Taken together, we interpret these results to indicate that different crabapple cultivars have different regulatory mechanisms in response to LTs, and the LT-response TFs mainly regulate anthocyanin biosynthetic upstream genes.

\section{Discussion}

Low temperature induces red pigment in foliage. Anthocyanins are beneficial for human health and there is great interest in developing strategies to increase their production in crops, and in understanding the factors that control their biosynthesis. Anthocyanin accumulation is developmentally controlled and is also affected by both biotic and abiotic factors, such as nutrients (nitrogen and phosphate), sucrose, wounding, pathogen infection, methyl jasmonate, water stress, and $\mathrm{pH}$, as well as ultraviolet, visible, and far-red light (Chalker-Scott, 1999; Dixon and Paiva 1995; Li et al., 1993). Temperature has also been reported to influence anthocyanin biosynthesis in many plant species, such as petunia (Petunia hybrida), rose (Rosa hybrida), red orange [Citrus sinensis (L.) Osbeck], grape [Vitis vinifera (L.) cv. Cabernet Sauvignon] and garlic (Allium sativum) (Dela et al., 2003; Lo Piero et al., 2005; Mori et al., 2007; Shvarts et al., 1997). In Arabidopsis thaliana, anthocyanin production is induced by LTs and reduced by high temperatures (Leyva et al., 1995; Rowan et al., 2009), whereas in apple, high temperatures have been shown to prevent the accumulation of cyanidin and UDP-sugars (Ban et al., 2009), resulting in a rapid reduction in anthocyanin levels (Steyn et al., 2004). In garlic (Allium sativum), LT conditions increase the synthesis of anthocyanins in the outer scale leaves of the bulbs at harvest time and this is preceded by an increase in the expression of putative phenylalanine ammonia lyase and uridine diphosphate glucose (UDPG)-flavonoid glucosyl transferase genes (Dufoo-Hurtado et al., 2013).

The LT $\left(16{ }^{\circ} \mathrm{C}\right)$ treatment increased the anthocyanin accumulation in the leaves of the 'Royalty' and 'Prairifire' cultivars and the levels on day 8 were almost double to those on day 4 (Fig. 2C). Anthocyanins and
A

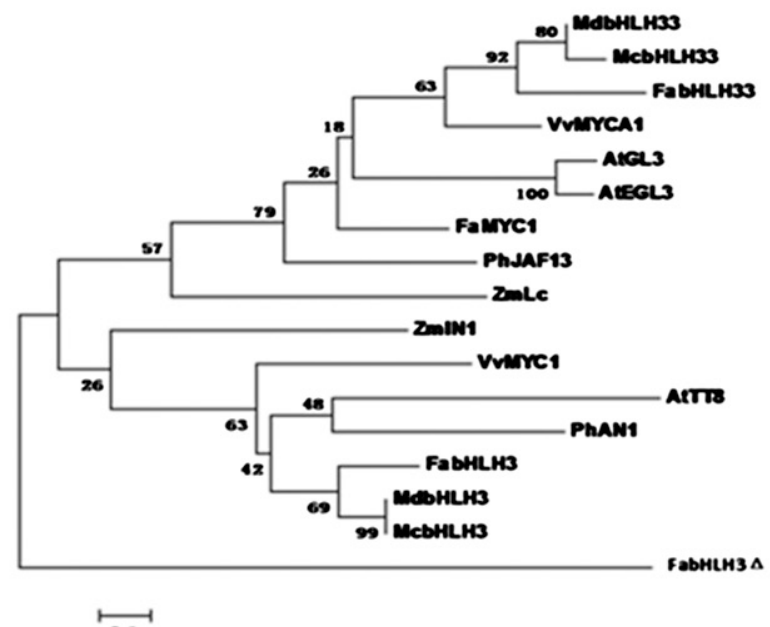

B
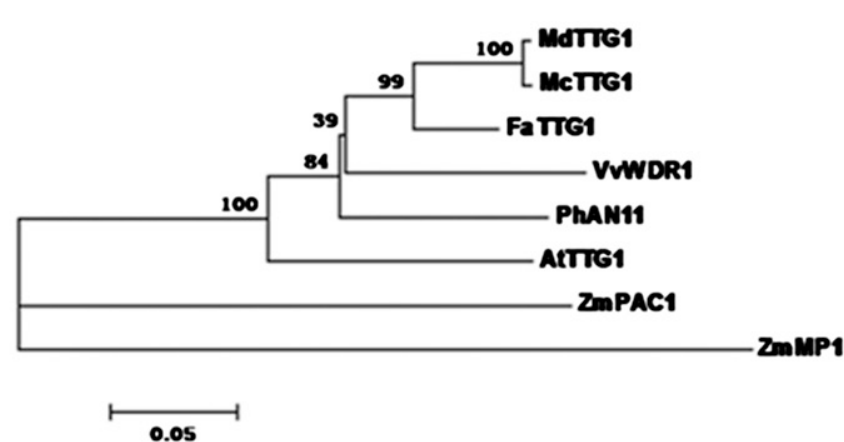

Fig. 4. Phylogenetic analysis of McbHLH3, McbHLH33, and McTTG1 with other known basic helix-loop-helix (bHLH) and TTG genes involved in the regulation of anthocyanin biosynthesis. Phylogenetic and molecular evolutionary analysis was conducted using MEGA version 5.10 (using the minimum evolution phylogeny test and 1000 bootstrap replicates). (A) Phylogenetic tree of McbHLH3 and McbHLH33 with other known bHLH proteins. (B) Phylogenetic tree of McTTG1 with other known TTG proteins. The GenBank accession numbers of the proteins are shown in Figure 3. 

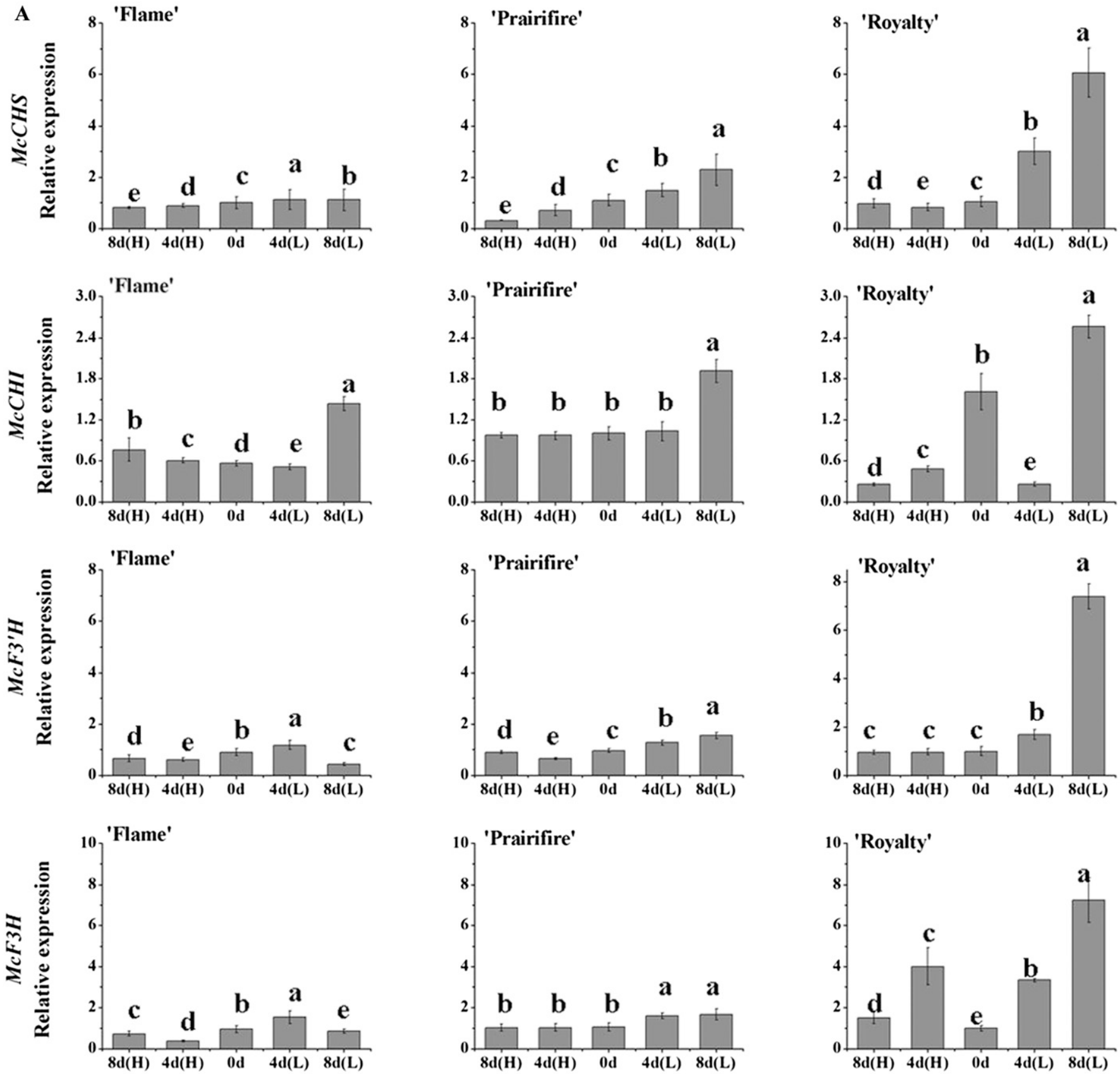

Fig. 5. Expression profiling of Malus crabapple anthocyanin biosynthesis structural and regulatory genes during temperature treatment in three different cultivars. (A) The expression level of early-stage anthocyanin biosynthetic genes. (B) The expression level of late-stage anthocyanin biosynthetic genes. (C) The expression level of anthocyanin biosynthesis regulatory genes.

flavones were present at significantly lower concentrations, or were not detectable, in 'Flame' leaves compared with those of the other two cultivars. Previously results showed a 23-bp repeat motif in the upstream regulatory region of alleles of MdMYB10 found only in red-fleshed apples (Espley et al., 2009), and the repeat motif was also found in crabapple (Tian et al., 2015), leading us to hypothesize that the anthocyanins accumulation of $\mathrm{LT}$ responses required the important cis-element in McMYB10 promoter.

Flavonols play a role in protection against ultraviolet radiation and other environment stress in plants (Li et al., 1993). The flavonol content in 'Royalty' and 'Prairifire' cultivars increased following the LT treatment, indicating that flavonols may be involved in cold resistance processes in crabapple (Fig. 2C). Moreover, we deduced that the increased flavonol may be tolerant to chilling in these color-leafed crabapple cultivars. As global warming progresses, temperature plays a modulating role and triggers the visible progress of phenology, such as leaf coloration, in many species (Körner and Basler, 2010). Previous studies have shown that leaf coloration in Japanese maple has been markedly delayed as a consequence of global warming over the past half century (Ibáñez et al., 2010). In Europe and Japan, leaf color changes have shown a delay of 0.3 $1.6 \mathrm{~d}$ per decade, whereas the length of growing season has increased by up to $3.6 \mathrm{~d}$ per decade over the past 50 years (Matsumoto et al., 2003; Menzel 2000; Menzel and Fabian 1999). All these plant phenological changes are highly correlated with temperature changes. So we conclude that LTs are important in crabapple leaf color development, and in the context of urban landscaping, this species may be more appropriate for use in the north hemisphere that experiences lower temperatures.

Low temperature promotes the expressions of anthocyanin biosynthesis genes. Many aspects of anthocyanin biosynthesis and its 

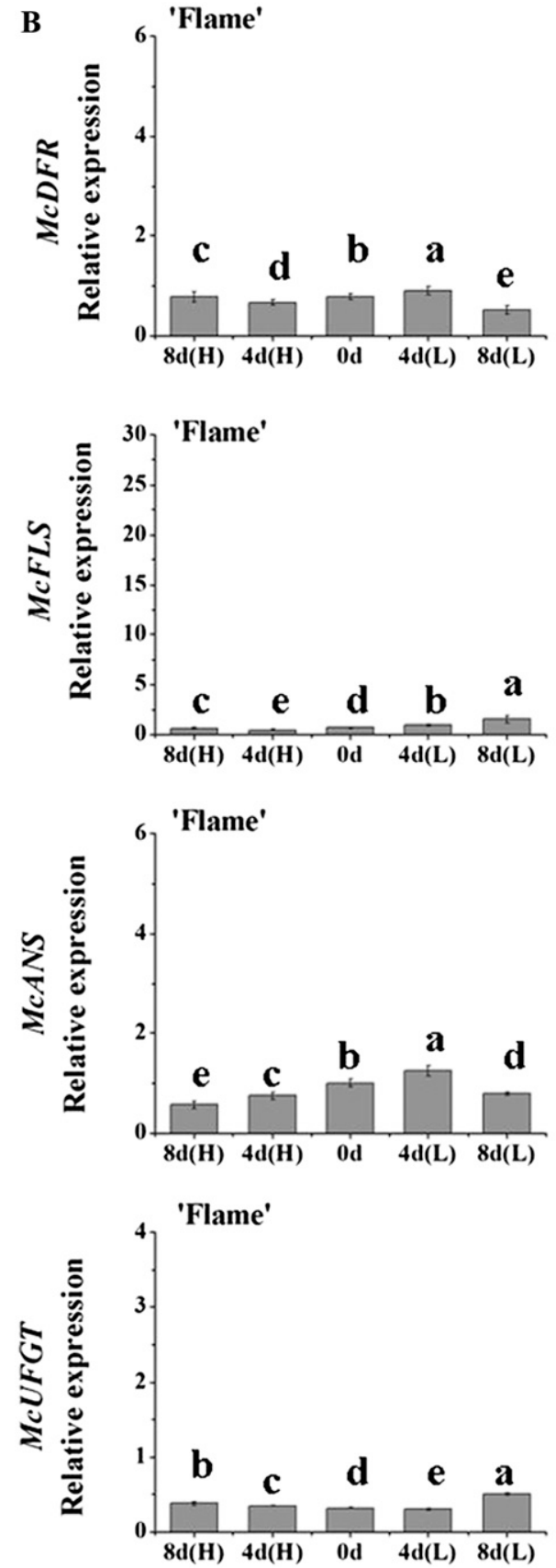
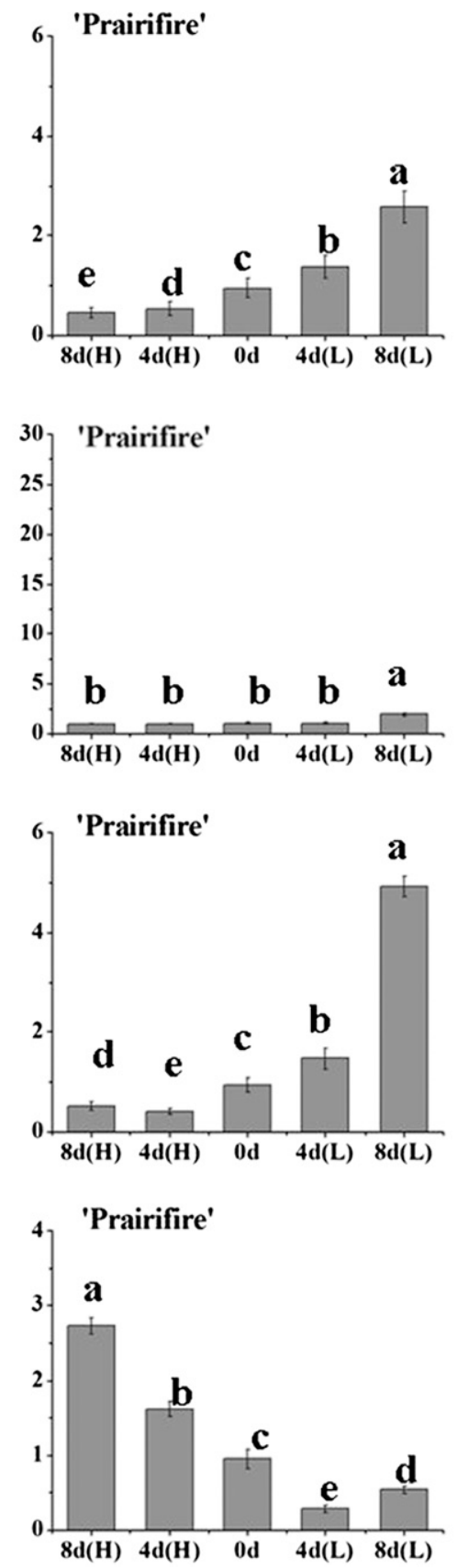
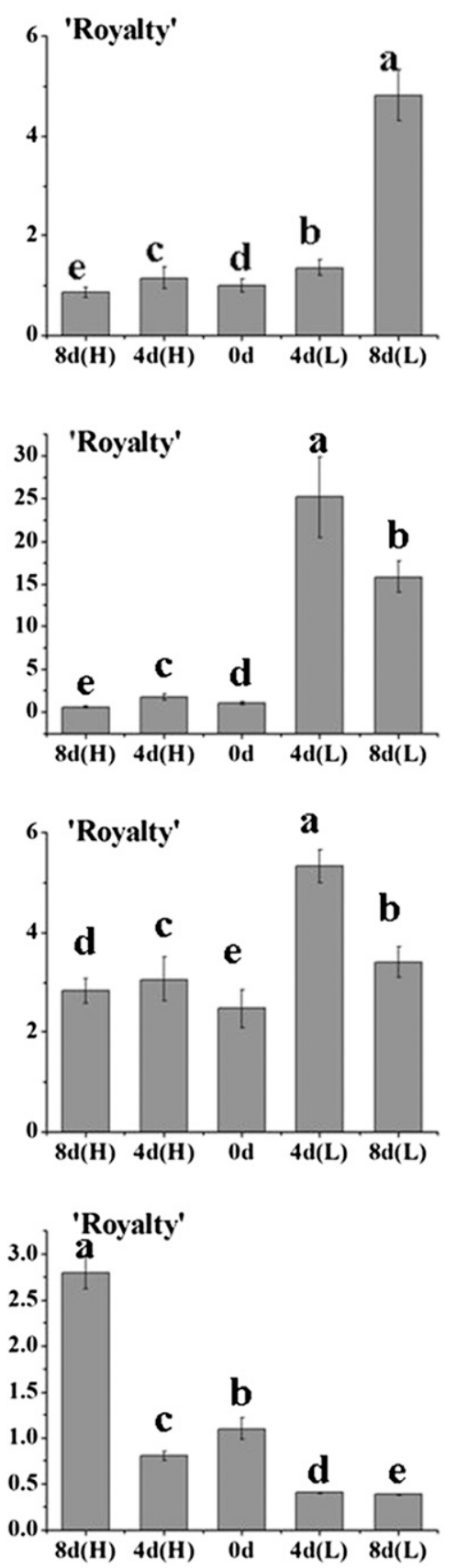

Fig. 5. (continued)

regulation have been studied (Allan et al., 2008) and it has been shown that phenylalanine ammonium lyase-1 $(P A L)$ and chalcone synthase $(C H S)$ mRNAs accumulate in a lightdependent manner in Arabidopsis thaliana leaves when exposed to LT (Leyva et al., 1995). In red orange, anthocyanins accumulate in vesicles and the expression of $P A L$, $C H S$, dihydroflavonol 4-reductase $(D F R)$, and UDPG-flavonoid 3-O-glucosyl transferase (UFGT) is strongly induced when stored at $4{ }^{\circ} \mathrm{C}$ (Lo-Piero et al., 2005). Similarly, in apple (Malus domestica), the expression of $C H S$, anthocyanidin synthase (ANS), and $U F G T$ was reported to be enhanced along with the accumulation of anthocyanins in the fruit skin following LT treatments (Ubi et al., 2006). We note that our results showed some differences from previous studies. Specifically, the expression data suggest that early anthocyanin biosynthetic genes $(M c C H S, M c F 3 H$, and $M c D F R$ ) are involved in the low-temperature induction of anthocyanin biosynthesis in crabapple leaves and contribute to the elevation in leaf anthocyanin levels. In contrast, the expression patterns of McANS and McUFGT suggest no such association with anthocyanin accumulation in response to $\mathrm{LT}$ treatments. This result distinguishes crabapple leaves from previous studies of other species and organs. Meanwhile, in our previous study, low environmental $\mathrm{pH}$ values are able to promote anthocyanins accumulation in colored-leaves crabapple cultivars by activating the expression of late anthocyanin biosynthetic genes (McANS and McUFGT). Thus, we speculate that the altered soil environment can trigger red foliage coloration by activating different stage of anthocyanin biosynthetic genes.

Upregulation of the anthocyanins pathway via an increased expression level of transcriptional activation complex. In our study, the expression of McMYB10, McbHLH3, McbHLH33, and McTTG1 was induced in crabapple leaves by the LT treatments, consistent with the observed increase in anthocyanin content (Figs. 2 and $5 \mathrm{C}$; Tables 2-4). In apple, the expression of $M d M Y B 10$ was reported to be downregulated 

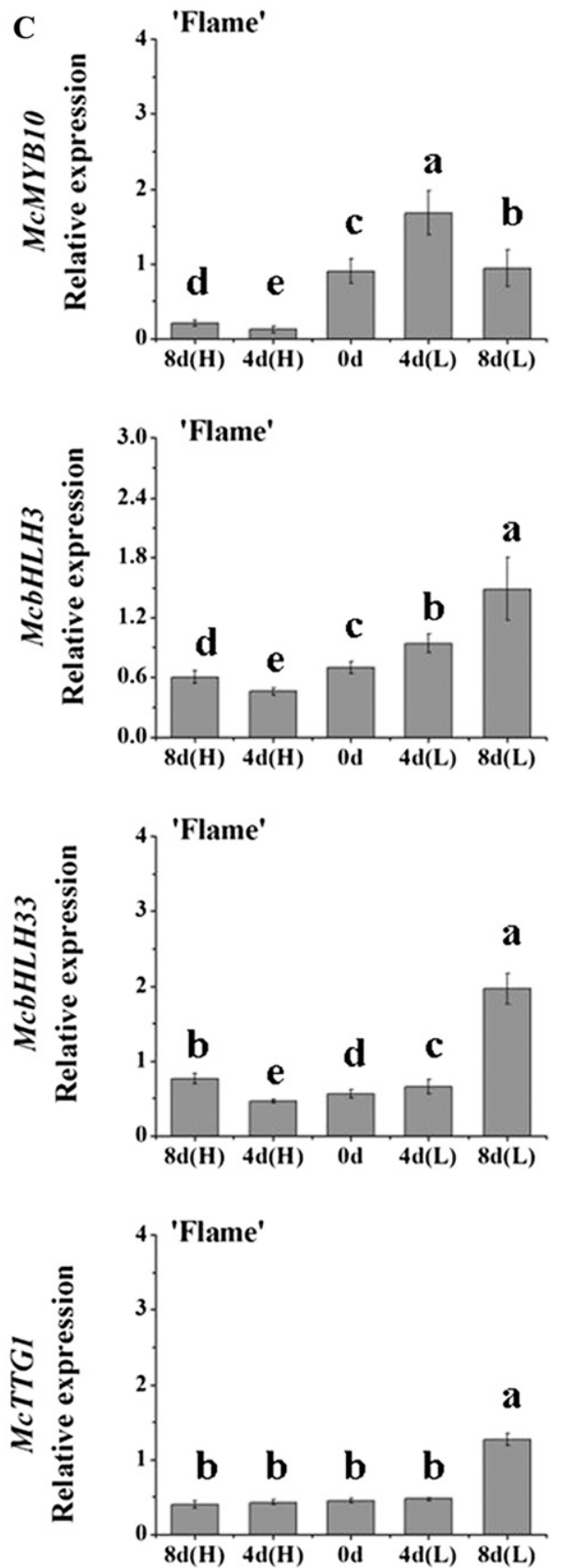
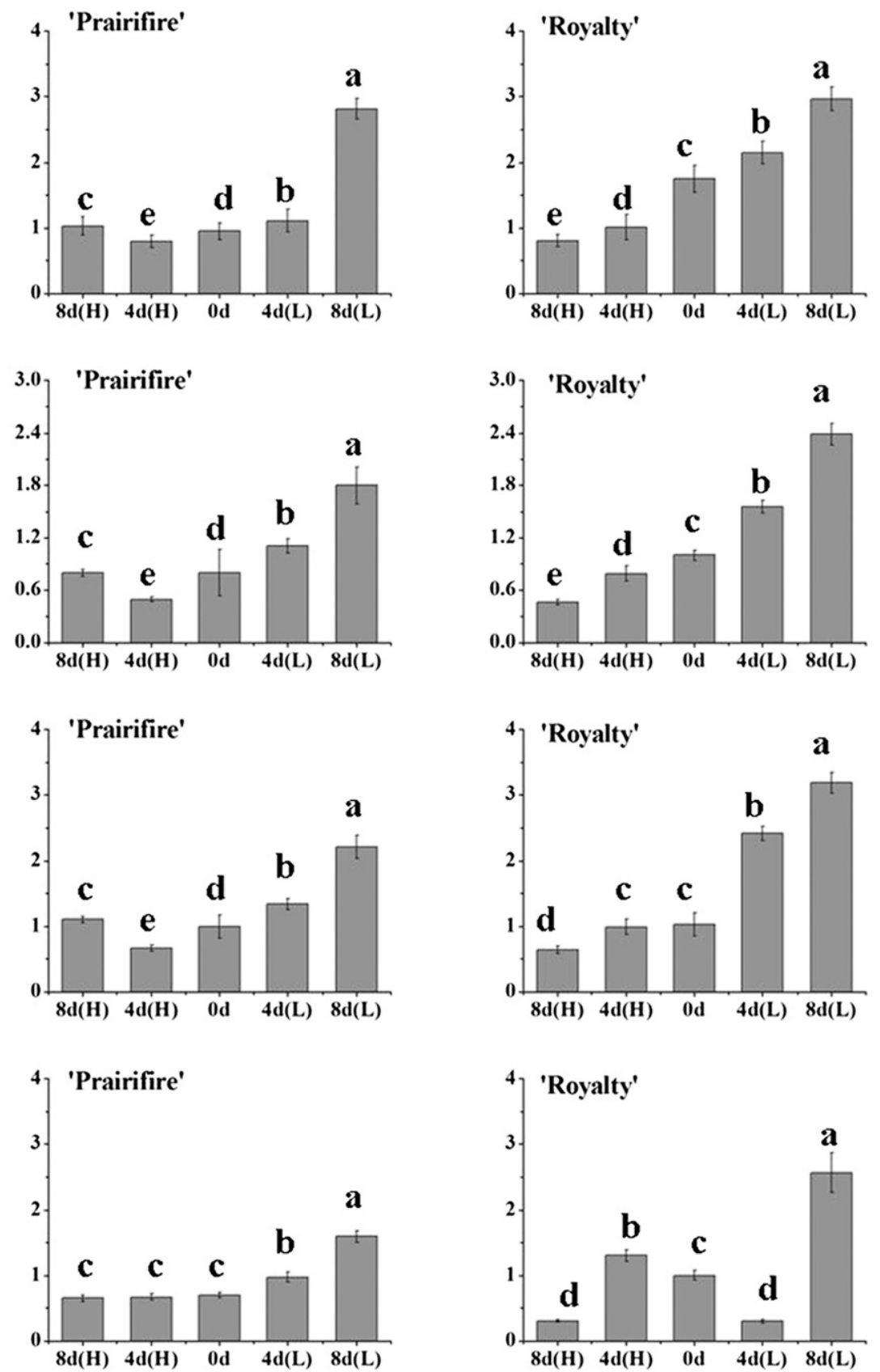
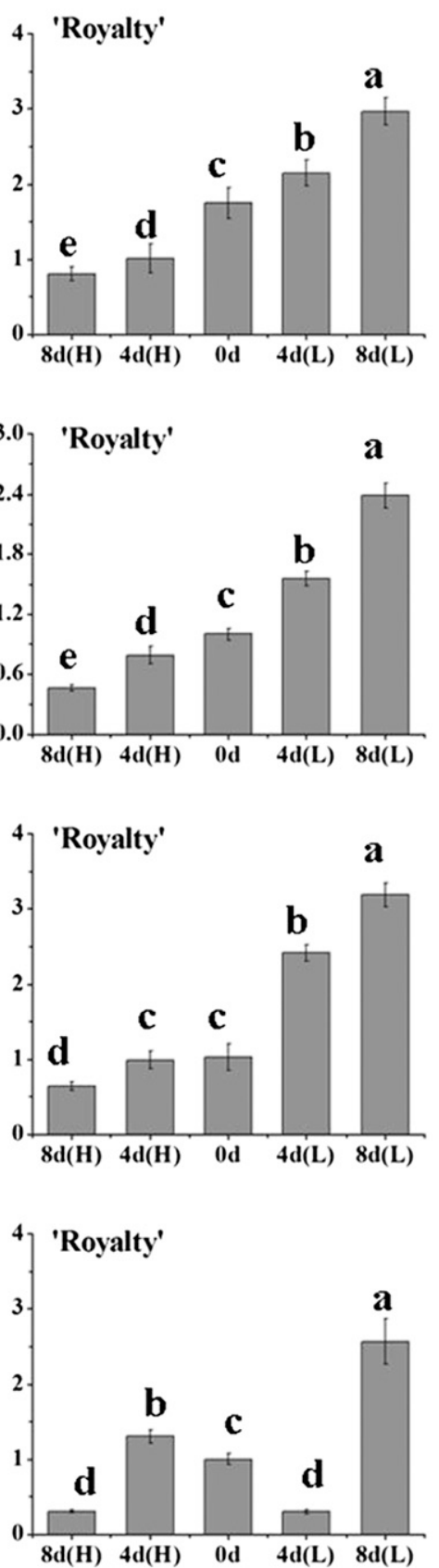

Fig. 5. (continued)

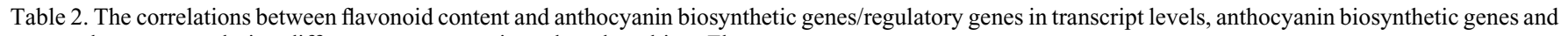
regulatory genes during different temperature in crabapple cultivar Flame.

\begin{tabular}{|c|c|c|c|c|c|c|c|c|c|c|c|c|c|c|}
\hline & Flavonol & Flavone & $\mathrm{McCHS}$ & $\mathrm{McCHI}$ & $M c F 3 H$ & $M c F 3^{\prime} H$ & $M c D F R$ & $M c F L S$ & $M c A N S$ & $M c U F G T$ & McMYB10 & McbHLH3 & McbHLH33 & McTTG1 \\
\hline Flavonol & 1 & -0.46 & 0.43 & -0.6 & $0.93 * *$ & $0.82 *$ & 0.79 & -0.06 & $0.85^{*}$ & -0.7 & 0.75 & -0.06 & -0.43 & -0.4 \\
\hline Flavone & -0.46 & 1 & 0.53 & $0.97 * *$ & -0.62 & -0.04 & $-0.82 *$ & $0.90^{*}$ & -0.17 & $0.93 * *$ & 0.15 & $0.90 *$ & $0.98 * *$ & $1.00 * *$ \\
\hline $\mathrm{McCHS}$ & 0.43 & 0.53 & 1 & 0.32 & 0.31 & 0.7 & -0.1 & 0.79 & 0.74 & 0.19 & $0.88 *$ & 0.79 & 0.5 & 0.59 \\
\hline $\mathrm{McCHI}$ & -0.6 & $0.97 * *$ & 0.32 & 1 & -0.74 & -0.17 & $-0.84 *$ & $0.81^{*}$ & -0.39 & $0.99 * *$ & -0.04 & $0.81 *$ & $0.98 * *$ & $0.95 * *$ \\
\hline$M c F 3 H$ & $0.93 * *$ & -0.62 & 0.31 & -0.74 & 1 & 0.78 & $0.90^{*}$ & -0.22 & $0.84 *$ & $-0.83 *$ & 0.68 & -0.22 & -0.58 & -0.56 \\
\hline$M c F 3^{\prime} H$ & $0.82 *$ & -0.04 & 0.7 & -0.17 & 0.78 & 1 & 0.59 & 0.4 & $0.83^{*}$ & -0.31 & $0.95^{* *}$ & 0.4 & 0.04 & 0.02 \\
\hline$M c D F R$ & 0.79 & $-0.82 *$ & -0.1 & $-0.84 *$ & $0.90 *$ & 0.59 & 1 & -0.5 & 0.52 & $-0.88 *$ & -0.37 & -0.5 & -0.74 & -0.79 \\
\hline$M c F L S$ & -0.06 & $0.90 *$ & 0.79 & $0.81 *$ & -0.22 & 0.4 & -0.5 & 1 & 0.2 & 0.72 & 0.55 & $1.00 * *$ & $0.92 * *$ & $0.92 * *$ \\
\hline$M c A N S$ & $0.85^{*}$ & -0.17 & 0.74 & -0.39 & $0.84 *$ & $0.83 *$ & 0.52 & 0.2 & 1 & -0.52 & $0.90 *$ & 0.2 & -0.2 & -0.1 \\
\hline$M c U F G T$ & -0.7 & $0.93 * *$ & 0.19 & $0.99 * *$ & $-0.83^{*}$ & -0.31 & $-0.88 *$ & 0.72 & -0.52 & 1 & -0.19 & 0.72 & $0.93 * *$ & $0.90 *$ \\
\hline МcMYB10 & 0.75 & 0.15 & $0.88^{*}$ & -0.04 & 0.68 & $0.95 * *$ & -0.37 & 0.55 & $0.90 *$ & -0.19 & 1 & 0.55 & 0.17 & 0.22 \\
\hline McbHLH3 & -0.06 & $0.90 *$ & 0.79 & $0.81 *$ & -0.22 & 0.4 & -0.5 & $1.00 * *$ & 0.2 & 0.72 & 0.55 & 1 & $0.92 * *$ & $0.92 * *$ \\
\hline McbHLH33 & -0.43 & $0.98 * *$ & 0.5 & $0.98 * *$ & -0.58 & 0.04 & -0.74 & $0.92 * *$ & -0.2 & $0.93 * *$ & 0.17 & $0.92 * *$ & 1 & $0.98 * *$ \\
\hline McTTG1 & -0.4 & $1.00 * *$ & 0.59 & $0.95 * *$ & -0.56 & 0.02 & -0.79 & $0.92 * *$ & -0.1 & $0.90 *$ & 0.22 & $0.92 * *$ & $0.98 * *$ & 1 \\
\hline
\end{tabular}

$* P<0.05, * * P<0.01$. 
Table 3. The correlations between flavonoid content and anthocyanin biosynthetic genes/regulatory genes in transcript levels, anthocyanin biosynthetic genes and regulatory genes during different temperature in crabapple cultivar Prairifire.

\begin{tabular}{|c|c|c|c|c|c|c|c|c|c|c|c|c|c|c|c|}
\hline & nthoc & & lav & $\mathrm{McCH}$ & $\mathrm{McCH}$ & $M c F 3$ & $M c F 3^{\prime}$ & $M c D F I$ & $M c F L$ & $M c A \Lambda$ & IcUF & $\{c M Y \backslash$ & $1 c b H L$ & IcbHLH & ИсTTG 1 \\
\hline Anthocyanins & 1 & 0.31 & $0.83 *$ & $0.98 * *$ & $0.83^{*}$ & $0.83^{*}$ & 0.77 & $0.94 * *$ & $0.83 *$ & $0.89 *$ & $-0.83 *$ & $0.81^{*}$ & $0.84^{*}$ & 0.8 & $0.88^{*}$ \\
\hline Flavonol & 0.31 & 1 & -0.12 & 0.14 & 0.15 & 0.16 & -0.18 & 0.16 & 0.15 & 0.16 & -0.09 & 0.15 & 0.16 & 0.16 & 0.03 \\
\hline Flavone & $0.83^{*}$ & -0.12 & 1 & $0.92 * *$ & $0.88^{*}$ & $0.93 * *$ & $0.96^{* *}$ & $0.95^{* *}$ & $0.88 *$ & $0.93 * *$ & -0.65 & $0.90^{*}$ & $0.95 * *$ & $0.93 * *$ & $0.98 * *$ \\
\hline $\mathrm{McCHS}$ & $0.98 * *$ & 0.14 & $0.92 * *$ & 1 & $0.85^{*}$ & $0.90 *$ & $0.89^{*}$ & $0.98 * *$ & $0.85^{*}$ & $0.91 *$ & $-0.84 *$ & $0.84 *$ & $0.89^{*}$ & $0.86^{*}$ & $0.93 * *$ \\
\hline $\mathrm{McCHI}$ & $0.83^{*}$ & 0.15 & $0.88^{*}$ & $0.85^{*}$ & 1 & 0.81 & 0.72 & $0.93 * *$ & $1.00 * *$ & $0.99 * *$ & -0.44 & $0.99 * *$ & $0.92 * *$ & $0.93 * *$ & $0.96^{* *}$ \\
\hline$M c F 3 H$ & $0.83 *$ & 0.16 & $0.93 * *$ & $0.90^{*}$ & 0.81 & 1 & $0.92^{* *}$ & $0.94 * *$ & 0.81 & $0.89 *$ & -0.67 & $0.85^{*}$ & $0.97 * *$ & $0.95^{* *}$ & $0.91 *$ \\
\hline$M c F 3^{\prime} H$ & 0.77 & -0.18 & $0.96 * *$ & $0.89^{*}$ & 0.72 & $0.92 * *$ & 1 & $0.89 *$ & 0.72 & 0.81 & -0.76 & 0.74 & $0.87^{*}$ & $0.84^{*}$ & $0.88 *$ \\
\hline$M c D F R$ & $0.94 * *$ & 0.16 & $0.95 * *$ & $0.98^{* *}$ & $0.93 * *$ & $0.94 * *$ & $0.89 *$ & 1 & $0.93 * *$ & $0.98 * *$ & -0.71 & $0.93 * *$ & $0.96^{* *}$ & $0.94 * *$ & $0.98 * *$ \\
\hline$M c F L S$ & $0.83^{*}$ & 0.15 & $0.88^{*}$ & $0.85^{*}$ & $1.00 * *$ & 0.81 & 0.72 & $0.93 * *$ & 1 & $0.99 * *$ & -0.44 & $0.99 * *$ & $0.92 * *$ & $0.93 * *$ & $0.96^{* *}$ \\
\hline$M c A N S$ & $0.89 *$ & 0.16 & $0.93 * *$ & $0.91 *$ & $0.99 * *$ & $0.89 *$ & 0.81 & $0.98 * *$ & $0.99 * *$ & 1 & -0.55 & $0.99 * *$ & $0.96^{* *}$ & $0.96^{* *}$ & $0.99 * *$ \\
\hline McUFGT & $-0.83^{*}$ & -0.09 & -0.65 & $-0.84^{*}$ & -0.44 & -0.67 & -0.76 & -0.71 & -0.44 & -0.55 & 1 & -0.42 & -0.56 & -0.49 & -0.61 \\
\hline McMYB10 & $0.81^{*}$ & 0.15 & $0.90 *$ & $0.84^{*}$ & $0.99 * *$ & $0.85^{*}$ & 0.74 & $0.93 * *$ & $0.99 * *$ & $0.99 * *$ & -0.42 & 1 & $0.95 * *$ & $0.96^{* *}$ & $0.97 * *$ \\
\hline McbHLH3 & $0.84 *$ & 0.16 & $0.95 * *$ & $0.89 *$ & $0.92 * *$ & $0.97 * *$ & $0.87 *$ & $0.96 * *$ & $0.92 * *$ & $0.96 * *$ & -0.56 & $0.95^{* *}$ & 1 & $1.00 * *$ & $0.97 * *$ \\
\hline McbHLH33 & 0.8 & 0.16 & $0.93 * *$ & $0.86^{*}$ & $0.93 * *$ & $0.95 * *$ & $0.84 *$ & $0.94 * *$ & $0.93 * *$ & $0.96 * *$ & -0.49 & $0.96^{* *}$ & $1.00 * *$ & 1 & $0.96 * *$ \\
\hline McTTGI & $0.88^{*}$ & 0.03 & $0.98 * *$ & $0.93 * *$ & $0.96^{* *}$ & $0.91 *$ & $0.88^{*}$ & $0.98 * *$ & $0.96 * *$ & $0.99 * *$ & -0.61 & $0.97 * *$ & $0.97 * *$ & $0.96^{* *}$ & 1 \\
\hline
\end{tabular}

$* P<0.05, * * P<0.01$.

Table 4. The correlations between flavonoid content and anthocyanin biosynthetic genes/regulatory genes in transcript levels, anthocyanin biosynthetic genes and regulatory genes during different temperature in crabapple cultivar Royalty.

\begin{tabular}{|c|c|c|c|c|c|c|c|c|c|c|c|c|c|c|c|}
\hline & \multicolumn{15}{|c|}{ Anthocyanins Flavonol Flavone $M c C H S$ McCHI McF3H McF3' H McDFR McFLS McANS McUFGT McMYB10 McbHLH3 McbHLH33 McTTG } \\
\hline Anthocyanins & 1 & $0.91 *$ & $0.88^{*}$ & $0.96^{* *}$ & $0.83 *$ & $0.94 * *$ & 0.8 & $0.94 * *$ & 0.6 & 0.25 & -0.63 & $0.97 * *$ & $0.98^{* *}$ & $0.93 * *$ & 0.77 \\
\hline Flavonol & $0.91 *$ & 1 & $1.00 * *$ & $0.97 * *$ & 0.54 & $0.88^{*}$ & $0.88 *$ & $0.88^{*}$ & 0.8 & 0.55 & -0.65 & $0.88^{*}$ & $0.95 * *$ & $0.98 * *$ & 0.62 \\
\hline Flavone & $0.88 *$ & $1.00^{* *}$ & 1 & $0.95 * *$ & 0.5 & $0.85 *$ & $0.88^{*}$ & $0.85^{*}$ & $0.83^{*}$ & 0.6 & -0.7 & $0.87^{*}$ & $0.95 * *$ & $0.98 * *$ & 0.59 \\
\hline $\mathrm{McCHS}$ & $0.96 * *$ & $0.97 * *$ & $0.95^{* *}$ & 1 & 0.69 & $0.95 * *$ & $0.86^{*}$ & $0.95 * *$ & 0.69 & 0.38 & -0.56 & $0.92 *$ & $0.97 * *$ & $0.96^{* *}$ & 0.7 \\
\hline $\mathrm{McCHI}$ & $0.83^{*}$ & 0.54 & 0.5 & 0.69 & 1 & 0.81 & 0.56 & $0.81^{*}$ & 0.08 & -0.3 & -0.42 & 0.75 & 0.72 & 0.57 & $0.87^{*}$ \\
\hline$M c F 3 H$ & $0.94 * *$ & $0.88 *$ & $0.85 *$ & $0.95 * *$ & 0.81 & 1 & $0.88^{*}$ & $1.00 * *$ & 0.45 & 0.1 & -0.46 & $0.83^{*}$ & $0.90^{*}$ & $0.85^{*}$ & $0.85^{*}$ \\
\hline$M c F 3^{\prime} H$ & 0.8 & $0.88 *$ & $0.88 *$ & $0.86^{*}$ & 0.56 & $0.88^{*}$ & 1 & $0.90^{*}$ & 0.51 & 0.27 & -0.62 & 0.69 & $0.83^{*}$ & $0.82 *$ & $0.82 *$ \\
\hline$M c D F R$ & $0.94 * *$ & $0.88^{*}$ & $0.85 *$ & $0.95 * *$ & $0.81 *$ & $1.00 * *$ & $0.90^{*}$ & 1 & 0.43 & 0.09 & -0.49 & $0.83 *$ & $0.90^{*}$ & $0.84 *$ & $0.88 *$ \\
\hline$M c F L S$ & 0.6 & 0.8 & $0.83 *$ & 0.69 & 0.08 & 0.45 & 0.51 & 0.43 & 1 & $0.92 * *$ & -0.64 & 0.72 & 0.73 & $0.84^{*}$ & 0.07 \\
\hline$M c A N S$ & 0.25 & 0.55 & 0.6 & 0.38 & -0.3 & 0.1 & 0.27 & 0.09 & $0.92 * *$ & 1 & -0.5 & 0.4 & 0.43 & 0.59 & -0.25 \\
\hline$M c U F G T$ & -0.63 & -0.65 & -0.7 & -0.56 & -0.42 & -0.46 & -0.62 & -0.49 & -0.64 & -0.5 & 1 & -0.73 & -0.74 & -0.72 & -0.51 \\
\hline МcMYBI0 & $0.97 * *$ & $0.88^{*}$ & $0.87^{*}$ & $0.92 *$ & 0.75 & $0.83 *$ & 0.69 & $0.83^{*}$ & 0.72 & 0.4 & -0.73 & 1 & $0.98 * *$ & $0.94 * *$ & 0.63 \\
\hline McbHLH3 & $0.98 * *$ & $0.95 * *$ & $0.95^{* *}$ & $0.97 * *$ & 0.72 & $0.90^{*}$ & $0.83^{*}$ & $0.90 *$ & 0.73 & 0.43 & -0.74 & $0.98 * *$ & 1 & $0.98^{* *}$ & 0.7 \\
\hline McbHLH33 & $0.93 * *$ & $0.98 * *$ & $0.98 * *$ & $0.96^{* *}$ & 0.57 & $0.85 *$ & $0.82 *$ & $0.84 *$ & $0.84 *$ & 0.59 & -0.72 & $0.94 * *$ & $0.98 * *$ & 1 & 0.58 \\
\hline McTTGI & 0.77 & 0.62 & 0.59 & 0.7 & $0.87 *$ & $0.85 *$ & $0.82 *$ & $0.88^{*}$ & 0.07 & -0.25 & -0.51 & 0.63 & 0.7 & 0.58 & 1 \\
\hline
\end{tabular}

$* P<0.05, * * P<0.01$.

in fruits grown under natural or artificial hot temperate conditions (Lin-Wang et al., 2011). Moreover, it was shown that higher temperatures reduced $\mathrm{MdbHLH3}$ and MbHLH33 expression (Espley et al., 2007), whereas MdbHLH300, but not MdbHLH3 or MdbHLH33, were downregulated in fruits grown in a hot climate compared with those cultivated in a temperate climate (Lin-Wang et al., 2011). Other results have also indicated that MdbHLH3 expression is also induced by LTs at both the transcriptional and translational levels (Xie et al., 2012). The results presented here provide additional evidence that the MBW complex is involved in responses to environmental factors.

Interestingly, McbHLH3, McbHLH33, and McTTG1 were expressed at almost the same level in the green-leaf cultivar Flame and the red-leaf cultivar Royalty under LTs, but anthocyanins content were not detectable in 'Flame' leaves. We propose that these three TFs are responsible for the biosynthesis of flavonol in the green-leaf cultivar, in accordance with the observation that flavonol levels do not change during the early stage of the LT treatment in 'Flame'. In addition, the lower expression level of McMYB10 in 'Flame' may explain why the green-leaf cultivars did not produce anthocyanins at $16^{\circ} \mathrm{C}$ (Fig. 5C). The regulation of anthocyanins accumulation under LT through the alteration of the expression of members of the MBW complex may be caused by several processes. There may be direct temperature-induced changes in activity of the promoter of MYB10 (Espley et al., 2009), or the MBW complex may be a downstream component of the cold signaling pathway. It was recently hypothesized that plants recruit the MBW complex to regulate anthocyanins' biosynthesis in response to environmental temperatures not only at the transcriptional and translational levels but also at the posttranslational level (Xie et al., 2012).

\section{Conclusion}

Anthocyanins are effective and widespread protection factors in plants, and are beneficial to human health, as well as endowing plants with an attractive color, which is of commercial importance (Davies et al., 2003). Our results indicate that the MBW complex plays a key role in the regulation of anthocyanin biosynthesis under LT (Fig. 6). Moreover, we suggest that this information may be used to help optimize anthocyanin content or improve organ coloration in response to environmental temperatures, particularly in the context of global warming.

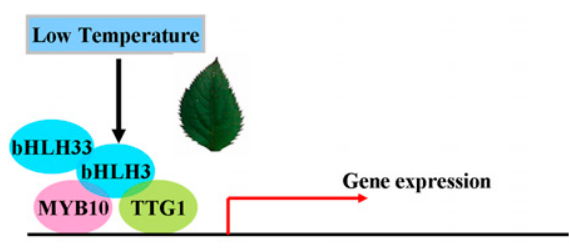

McCHS, McF3H, McF3'H, McDFR

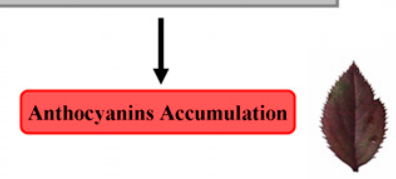

Fig. 6. A model for R2R3-MYB/basic helix-loophelix (bHLH)/WD40 (MBW)-induced anthocyanins accumulation under low temperature.

\section{Literature Cited}

Allan, A.C., R.P. Hellens, and W.A. Laing. 2008. MYB transcription factors that colour our fruit. Trends Plant Sci. 13:99-102.

Ban, Y., S. Kondo, B.E. Ubi, C. Honda, H. Bessho, and T. Moriguchi. 2009. UDP-sugar biosynthetic pathway: Contribution to cyanidin 3 -galactoside biosynthesis in apple skin. Planta 230:871-881.

Baudry, A., M.A. Heim, B. Dubreucq, M. Caboche, B. Weisshaar, and L. Lepiniec. 2004. TT2, TT8, and TTG1 synergistically specify the expression of $B A N Y U L S$ and proanthocyanidin 
biosynthesis in Arabidopsis thaliana. Plant J. 39:366-380.

Boudet, A.M. 2007. Evolution and current status of research in phenolic compounds. Phytochemistry 68:2722-2735.

Chalker-Scott, L. 1999. Environmental significance of anthocyanin in plant stress responses. Photochem. Photobiol. 70:1-9.

Chomczynski, P. and N. Sacchi. 2006. The singlestep method of RNA isolation by acid guanidinium thiocyanate-phenol-chloroform extraction: Twenty-something years on. Nat. Protoc. 1:581-585.

Davies, K.M., K.E. Schwinn, S.C. Derole, D.G. Manson, D.H. Lewis, S.J. Bloor, and J.M. Bradley. 2003. Enhancing anthocyanin production by altering competition for substrate between flavonol synthase and dihydroflavonol 4-reductase. Euphytica 131:259-268.

Dela, G., E. Or, R. Ovadia, A. Nissim-Levi, D. Weiss, and M. Oren-Shamir. 2003. Changes in anthocyanin concentration and composition in 'Jaguar' rose flowers due to transient high-temperature conditions. Plant Sci. 164: 333-340.

Deluc, L. 2006. Characterization of a grapevine R2R3-MYB transcription factor that regulates the phenylpropanoid pathway. Plant Physiol. 140:499-511.

Dixon, R.A. and N.L. Paiva. 1995. Stress-induced phenylpropanoid metabolism. Plant Cell 7:10851097.

Dufoo-Hurtado, M.D., K.G. Zavala-Gutieìrrez, C.M. Cao, L. Cisneros-Zevallos, R.G. GuevaraGonzailez, I. Torres-Pacheco, M.E. VaìzquezBarrios, D.M. Rivera-Pastrana, and E.M. Mercado-Silva. 2013. Low-temperature conditioning of "Seed" cloves enhances the expression of phenolic metabolism related genes and anthocyanin content in 'Coreano' Garlic (Allium sativum) during plant development. J. Agr. Food Chem. 61:10439-10446.

Espley, R.V., C. Brendolise, D. Chagne, S. KuttyAmma, S. Green, R. Volz, J. Putterill, H.J. Schouten, S.E. Gardiner, R.P. Hellens, and A. C. Allan. 2009. Multiple repeats of a promoter segment causes transcription factor autoregulation in red apples. Plant Cell 21:168-183.

Espley, R.V., R.P. Hellens, J. Putterill, D.E. Stevenson, S. Kutty-Amma, and A.C. Allan. 2007. Red colouration in apple fruit is due to the activity of the MYB transcription factor, MdMYB10. Plant J. 49:414-427.

Feller, A., K. Machemer, E.L. Braun, and E. Grotewold. 2011. Evolutionary and comparative analysis of MYB and bHLH plant transcription factors. Plant J. 66:94-116.

Heim, M.A., M. Jakoby, M. Werber, C. Martin, B. Weisshaar, and P.C. Bailey. 2003. The basic helixloop-helix transcription factor family in plants: A genome-wide study of protein structure and functional diversity. Mol. Biol. Evol. 20:735-747.

Henry-Kirk, R.A., T.K. McGhie, C.M. Andre, R.P. Hellens, and A.C. Allan. 2012. Transcriptional analysis of apple fruit proanthocyanidin biosynthesis. J. Expt. Bot. 63:5437-5450.

Hichri, I., F. Barrieu, J. Bogs, C. Kappe, S. Delrot, and V. Lauvergeat. 2011. Recent advances in the transcriptional regulation of the flavonoid biosynthetic pathway. J. Expt. Bot. 62:24652483.

Ibáñez, I., R.B. Primack, A.J. Miller-Rushing, E. Ellwood, H. Higuchi, S.D. Lee, H. Kobori, and J.A. Silander. 2010. Forecasting phenology under global warming. Philos. Trans. R. Soc. Lond. B Biol. Sci. 365:3247-3260.

Jiang, R., J. Tian, T.T. Song, J. Zhang, and Y.C. Yao. 2014. The Malus crabapple transcription factor McMYB10 regulates anthocyanin biosynthesis during petal coloration. Sci. Hort. 166:42-49.

Koes, R., W. Verweij, and F. Quattrocchio. 2005. Flavonoids: A colorful model for the regulation and evolution of biochemical pathways. Trends Plant Sci. 10:236-242.

Körner, C. and D. Basler. 2010. Phenology under global warming. Science 327:1461.

Kumar, S., K. Tamura, and M. Nei. 2004. MEGA3: Integrated software for molecular evolutionary genetics analysis and sequence alignment. Brief Bioinform. 5:150-163.

Lo-Piero, A.R., I. Puglisi, P. Rapisarda, and G. Petrone. 2005. Anthocyanins accumulation and related gene expression in red orange fruit induced by low temperature storage. J. Agr. Food Chem. 53:9083-9088.

Leyva, A., J.A. Jarillo, J. Salinas, and J.M. MartinezZapater. 1995. Low temperature induces the accumulation of phenylalanine ammonia-lyase and chalcone synthase mRNAs of Arabidopsis thaliana in a light-dependent manner. Plant Physiol. 108:39-46.

Li, J., T.M. Ou-Lee, R. Raba, R.G. Amundson, and R.L. Last. 1993. Arabidopsis flavonoid mutants are hypersensitive to UV-B irradiation. Plant Cell 5:171-179.

Lin-Wang, K., D. Micheletti, J. Palmer, R. Volz, L. Lozano, R. Espley, R.P. Hellens, D. Chagne, D.D. Rowan, M. Troggio, I. Iglesias, and A.C. Allan. 2011. High temperature reduces apple fruit colour via modulation of the anthocyanin regulatory complex. Plant Cell Environ. 34:1176-1190.

Martin, C. 2013. The interface between plant metabolic engineering and human health. Curr. Opin. Biotechnol. 24:344-353.

Matsumoto, K., T. Ohta, and T. Nakamura. 2003. Climate change and extension of the Ginkgo bioloba L. growing season in Japan. Glob. Change Biol. 9:1634-1642.

Menzel, A. 2000. Trends in phenological phases in Europe between 1951-1996. Intl. J. Biometeorol. 44:76-81.

Menzel, A. and P. Fabian. 1999. Growing season extended in Europe. Nature 397:659.

Moreau, C., M.J. Ambrose, L. Turner, L. Hill, T.H. Ellis, and J.M.I. Hofer. 2012. The $b$ gene of pea encodes a defective flavonoid 3',5' -hydroxylase, and confers pink flower color. Plant Physiol. 159:759-768

Mori, K., N. Goto-Yamamoto, M. Kitayama, and K. Hashizume. 2007. Loss of anthocyanin in red-wine grape under high temperature. J. Expt. Bot. 58:1935-1945.

Pfeiffer, J., C. Kühnel, J. Brandt, D. Duy, P.A. Nimal-Punyasiri, G. Forkmann, and T.C. Fischer. 2006. Biosynthesis of flavan 3-ols by leucoanthocyanidin 4-reductases and anthocyanidin reductases in leaves of grape (Vitis vinifera L.), apple (Malus $\times$ domestica Borkh.) and other crops. Plant Physiol. Biochem. 44: 323-334.

Rowan, D.D., M. Cao, K. Lin-Wang, J.M. Cooney, D.J. Jensen, P.T. Austin, M.B. Hunt, C. Norling, R.P. Hellens, R.J. Schaffer, and A.C. Allan. 2009. Environmental regulation of leaf colour in red 35S:PAP1 Arabidopsis thaliana. New Phytol. 182:102-115.

Schaart, J.G., C. Dubos, De La F.I. Romero, A.M. Houwelingen, and R.C. Vos. 2013. Identification and characterization of MYB-bHLH-WD40 regulatory complexes controlling proanthocyanidin biosynthesis in strawberry (Fragaria $x$ ananassa) fruits. New Phytol. 197:454-467.

Shvarts, M., A. Borochov, and D. Weiss. 1997. Low temperature enhances petunia flower pigmentation and induces chalcone synthase gene expression. Physiol. Plant. 99:67-72.

Steyn, W., S. Wand, D. Holcroft, and G. Jacobs. 2004. Red colour development and loss in pears. IX International Pear Symposium, Stellenbosch, South Africa. 671:79-85.

Tian, J., H.X. Shen, J. Zhang, T.T. Song, and Y.C. Yao. 2011. Characteristics of chalcone synthase promoters from different leaf-color Malus crabapple cultivars. Sci. Hort. 129: 449-458.

Tian, J., Z. Peng, J. Zhang, T.T. Song, H.H. Wan, M.L. Zhang, and Y.C. Yao. 2015. McMYB10 regulates coloration via activating $M c F 3^{\prime} H$ and later structural genes in ever red leaf crabapple. Plant Biotechnol. J., doi:10.1111/pbi.12331.

Ubi, B.E., C. Honda, H. Bessho, S. Kondo, M. Wada, S. Kobayashi, and T. Moriguchi. 2006. Expression analysis of anthocyanin biosynthetic genes in apple skin: Effect of UV-B and temperature. Plant Sci. 170:571-578.

Winkel-Shirley, B. 2001. Flavonoid biosynthesis. A colorful model for genetics, biochemistry, cell biology, and biotechnology. Plant Physiol. 126:485-493.

Xie, X.B., S. Li, R.F. Zhang, J. Zhao, Y.C. Chen, Q. Zhao, Y.X. Yao, C.X. You, X.S. Zhang, and Y.J. Hao. 2012. The bHLH transcription factor MdbHLH3 promotes anthocyanin accumulation and fruit colouration in response to low temperature in apples. Plant Cell Environ. 35:1884-1897.

Zhang, Y.C., J. Zhang, T.T. Song, J.Y. Li, J. Tian, K.N. Jin, and Y.C. Yao. 2014. Low medium $\mathrm{pH}$ value enhances anthocyanin accumulation in Malus crabapple leaves. PLoS ONE 9:E97904. 